\title{
Clinical relevance of the borderline results of the Hybrid Capture 2 High-Risk HPV DNA assay with cervical samples collected in Specimen Transport Medium
}

\author{
Jerneja Varl'1,2, Urska Ivanus ${ }^{3}$, Ziva Pohar Marinsek ${ }^{4}$, Tine Jerman ${ }^{3}$, \\ Anja Ostrbenk Valencak ${ }^{5}$, Mario Poljak ${ }^{5}$, Veronika Kloboves Prevodnik ${ }^{4}$ \\ ${ }^{1}$ Department of Experimental Oncology, Institute of Oncology Ljubljana, Ljubljana, Slovenia \\ 2 University of Ljubljana, Faculty of Medicine, Ljubljana, Slovenia \\ ${ }^{3}$ ZORA National Cervical Cancer Screening Programme, Epidemiology and Cancer Registry, Institute of Oncology Ljubljana, \\ Ljubljana, Slovenia \\ ${ }^{4}$ Department of Cytopathology, Institute of Oncology Ljubljana, Ljubljana, Slovenia \\ ${ }^{5}$ Institute of Microbiology and Immunology, Faculty of Medicine, University of Ljubljana, Slovenia
}

Radiol Oncol 2019; 53(3): 316-322.

Received 10 July 2019

Accepted 6 August 2019

Correspondence to: Assoc. Prof. Veronika Kloboves Prevodnik, M.D., Ph.D., Department of Cytopathology, Institute of Oncology Ljubljana, Zaloska 2, SI-1000 Ljubljana, Slovenia. E-mail: vkloboves@onko-i.si

Disclosure: No potential conflicts of interest were disclosed.

Background. The Hybrid Capture 2 (HC2) High-Risk HPV DNA assay serves as a triage test in the Slovenian national cervical cancer screening programme ZORA. To improve the limited analytical accuracy of HC2 test results near the cut-off value (1.0 relative light units/cut-off (RLU/CO)), we follow an internal protocol of repeating the test on all samples with borderline results within the 0.7-2.0 RLU/CO interval. The aim of the study was (i) to determine the clinical relevance of HC2 test results within three different "grey zones" for samples stored in Specimen Transport Medium (STM) and (ii) to determine whether the current algorithm of retesting "grey zone" STM specimens with the HC2 assay is clinically relevant.

Patients and methods. The study included 594 women between 20 and 65 years of age. All participating women were referred for colposcopy, and in cases of abnormal results, biopsy was performed. We assessed the distribution of $\mathrm{HC} 2$ test results and the corresponding proportion of cervical intraepithelial neoplasia grade 2 or worse (CIN2+) lesions in three different "grey zones" (1.0-2.5, 0.4-4.0 and 0.7-2.0 RLU/CO), retested specimens with results within a 0.4-4.0 RLU/CO interval and calculated the sensitivity and specificity for $\mathrm{HC} 2$ at different RLU/CO values.

Results. The proportion of specimens within 1.0-2.5, 0.4-4.0 and 0.7-2.0 RLU/CO intervals was 3.9\%, $10.8 \%$ and $4.5 \%$, respectively. The proportion of CIN2+ lesions within these "grey zones" was $2.5 \%, 5.6 \%$ and $1.2 \%$, respectively. Retesting the samples did not detect any additional CIN2+ cases. Within the 1.0-2.5 RLU/CO interval, the sensitivity decreased from $93.8 \%$ to $91.4 \%$, while the specificity increased from $63.3 \%$ to $67.5 \%$; for the $0.4-4.0$ RLU/CO interval, the sensitivity decreased from $95.1 \%$ to $89.5 \%$, while the specificity increased from $56.8 \%$ to $69.4 \%$; and for the $0.7-2.0$ RLU/CO interval, the sensitivity remained nearly constant (94.4 vs. $93.2 \%$ ), while the specificity increased from $60.6 \%$ to $66.4 \%$. Conclusions. Our results show that retesting STM samples within the "grey zones" is not necessary. Retesting samples in the negative "grey zone" does not increase sensitivity, and retesting in the positive "grey zone" is not followed by a less intensive management of women, since these women are recalled regardless of the results of the retest. Furthermore, the majority of samples retain the original HC2 results after retest, and the number of CIN2+ lesions among women with "grey zone" HC2 results is low.

Key words: Hybrid Capture 2; HPV test; borderline results; grey zone; Specimen Transport Medium 


\section{Introduction}

The Slovenian national cervical cancer screening programme ZORA uses the Hybrid Capture II (HC2) assay (Qiagen, Hilden, Germany) as a triage test that stratifies women with low-grade cervical changes into those with high and low risks for developing cervical cancer. ${ }^{1}$ The results of the HC2 test are presented as relative light units/ cut-off (RLU/CO) values, and the cut-off value for a positive result is $1.0 \mathrm{RLU} / \mathrm{CO}^{2}$. Many studies have confirmed the high reproducibility of the HC2 test results both within and between laboratories. ${ }^{3-5}$ Nevertheless, some studies have noted that analytical accuracy is significantly lower in the vicinity of the cut-off value. . $^{3,46-8}$ Therefore, the manufacturer has published instructions for the further management of samples with borderline results that differ regarding which medium is used for sample collection. For PreservCyt specimens (Hologic Inc., Marlborough, United States), the manufacturer proposed the implementation of a borderline RLU/CO area called the "grey zone" in the range of 1.0-2.5 RLU/CO and recommended retesting the samples when results fall within this range. ${ }^{2}$ However, when storing the samples in the Specimen Transport Medium (STM) (Qiagen, Hilden, Germany), the manufacturer's instructions are different, recommending retesting only the samples with suspected HPV infection and those with HC2 results near but below the 1.0 RLU/CO value. Retesting can be performed with the $\mathrm{HC} 2$ test or using another method. ${ }^{2}$

Several authors ${ }^{9-16}$ have investigated the reproducibility and clinical significance of retesting PreservCyt samples with borderline HC2 results. However, we have not found a single study where the same problem was addressed for specimens collected in STM. A previous Slovenian study by Seme et al. ${ }^{6}$ evaluated the analytical accuracy of the results within 0.4-4.0 RLU/CO. Because these authors found poor reproducibility of $\mathrm{HC} 2$ within this range, they recommended that tests should be repeated by an alternative PCR-based method. At the Institute of Oncology Ljubljana, we adapted these instructions for our laboratory settings to repeat the test with the $\mathrm{HC} 2$ assay on specimens for which $\mathrm{HC} 2$ results fall within the 0.7-2.0 RLU/CO interval.

The aims of our study were (I) to determine the clinical relevance of the HC2 test results within three different "grey zones" for STM samples and (II) to determine whether the current algorithm for retesting "grey zone" STM specimens with the HC2 assay is clinically relevant.

\section{Patients and methods}

\author{
Study population
}

The study population included 596 women who participated in the Slovenian HPV self-sampling project L3-5512 from April 2014 to July 2016. The L3-5512 study protocol has been previously described. ${ }^{17}$ All women were referred to colposcopy where smears for high-risk HPV testing were obtained. The indications for colposcopy followed the Slovenian national guidelines, including highgrade cytology, HPV positive triage test after repeated low-grade cytology, positive HPV test for the surveillance of the women treated for highgrade intraepithelial lesion (HSIL) and a positive HPV test on self-sampling. Women with abnormal colposcopy underwent colposcopy-guided biopsy, followed by histological evaluation according to $\mathrm{WHO}$ recommendations. ${ }^{18}$ The reported high-grade histological outcomes within one year after colposcopy included cervical intraepithelial neoplasia grade 2 (CIN2), cervical intraepithelial neoplasia grade 3 (CIN3), squamous cell carcinoma, carcinoma with origin outside the cervix, cervical glandular intraepithelial neoplasia grade 2/adenocarcinoma in situ (CGIN2/AIS), vaginal intraepithelial neoplasia grade 3 (VAIN3) and vulvar intraepithelial neoplasia grade 3 (VIN3). All data were obtained from the Registry of the national screening programme ZORA.

The study was approved by the National Medical Ethics Committee at the Slovenian Ministry of Health (consents Nos. 155/03/13 and 136/04/14). All participating women provided written informed consent.

\section{HPV testing}

For the detection of high-risk HPV, we used the $\mathrm{HC} 2$ assay (Qiagen). The HC2 assay was performed according to the manufacturer's instructions, and the results were reported as positive or negative using 1.0 RLU/CO as the cut-off value. ${ }^{2}$ Briefly, the test is a nucleic acid hybridization assay with signal amplification using microplate chemiluminescence for the qualitative detection of 13 high-risk types of HPV DNA in cervical and vaginal specimens. ${ }^{2}$ The results were also interpreted according to the "grey zone" ranges proposed by the manufacturer for PreservCyt (1-2.5 RLU/CO ${ }^{2}$ ), Seme et al. for STM (0.4-4.0 RLU/CO $\left.{ }^{6}\right)$ and the Department of Cytopathology Institute of Oncology Ljubljana for STM (0.7-2.0 RLU/CO). Residual samples were stored in the freezer $\left(-30^{\circ} \mathrm{C}\right)$ after the denaturation 
step. If the results for a specimen were within the 0.4-4.0 RLU/CO range, then the HC2 assay was repeated. When the results of the retest differed from the original results, we reported the final result as inconclusive.

\section{Statistics}

The results are presented as ranges of RLU/CO values for the HPV test result; a proportion of HPV test results in a specific range for all $\mathrm{HPV}$ test results; a proportion of women with $\mathrm{CIN} 2 / 3+$ in a specific range for all women with $\mathrm{CIN} 2 / 3+$ in one year since colposcopy; and a risk for $\mathrm{CIN} 2 / 3+$ as a proportion of women with CIN2/3+ for all women in a specific range. Cohen's kappa with a $95 \%$ confidence interval (CI) was calculated as a measure of agreement between the original and retested HPV test results as a binary variable at a 1.0 RLU/CO cut-off value. Sensitivities and specificities were calculated with a 95\% CI at different RLU/CO cut-off values, and the ROC curve was plotted. All analyses were conducted with R v3.5.3. ${ }^{19}$

\section{Results}

\section{Study population}

Our final study group included 594 women after we excluded one woman who had undergone hys-

TABLE 1. Number of HPV test results according to "grey zones" proposed by the manufacturer (PreservCyt)', Seme et al. (STM)", and the Department of Cytopathology at Institute of Oncology Ljubljana (STM)"II

\begin{tabular}{lc}
\hline RLU/CO value I & N and \% women $\left(\mathrm{N}_{\text {tot }}=594\right)$ \\
\hline$<1.0$ & $283(47.6)$ \\
$1.0-2.5$ & $23(3.9)$ \\
$>2.5$ & $288(48.5)$ \\
\hline RLU/CO value ${ }^{\text {II }}$ & N and \% women (Tot. $\mathrm{N}=594)$ \\
\hline$<0.4$ & $253(42.6)$ \\
$0.4-0.99$ & $34(5.1)$ \\
$1.0-3.99$ & $277(46.6)$ \\
$\geq 4.0$ & N and \% women (Tot. N = 594) \\
\hline RLU/CO value ${ }^{\text {III }}$ & $271(45.6)$ \\
\hline$<0.7$ & $12(2.0)$ \\
$0.7-0.99$ & $15(2.5)$ \\
$1.0-1.99$ & $296(49.8)$ \\
$\geq 2.0$ &
\end{tabular}

$\mathrm{N}=$ number; $\mathrm{N}_{\text {tot }}=$ total number terectomy prior to colposcopy and another with missing data from colposcopy. Biopsy was performed in 352 women (59.3\%). Out of 594 women, $291(49.0 \%)$ were negative either on colposcopy (242) or on histology (49). A histologically confirmed low-grade intraepithelial lesion (LSIL) was diagnosed in 141 (23.7\%) women and CIN2+ was diagnosed in $162(27.3 \%)$ women. There were 48 CIN2, 102 CIN3, 1 VAIN3, 1 VIN3, 4 AIS and 6 squamous carcinomas.

\section{HPV test results based on different definitions for the "grey zone" range}

The number of HPV test results within and outside the three "grey zone" ranges are presented in Table 1. The proportion of samples located in 1.0-2.5 RLU/CO, 0.4-4.0 RLU/CO, and 0.7-2.0 RLU/CO was 3.9\% (23/594), 10.8\% (64/594), and $4.5 \%$ (27/594), respectively.

\section{HPV test results for retested samples within the 0.4-4.0 RLU/CO range}

All 64 samples with results between 0.4-4.0 RLU/ $\mathrm{CO}$ values were retested. In 8/64 (12.5\%) retested samples, the results differed from the original results (Figure 1). Six samples for which the results changed from positive to negative originated from patients without CIN2+ lesions, while the seventh case was obtained from a patient with CIN3+ diagnosis. The sample for which the result changed from negative to positive was from a patient without a CIN2+ diagnosis. Kappa agreement between the results before and after retesting was 0.75 (95\% CI: 0.59-0.91). Retesting samples with results within the 0.4-4.0 RLU/CO range did not detect any additional CIN2+ cases.

\section{Detection of $\mathrm{CIN} 2+$ and $\mathrm{CIN} 3+$ at different ranges of RLU/CO values}

The distribution of women with a CIN2+/3+ diagnosis and the risk for CIN2+/3+ based on the RLU/CO values of their HPV test results are presented in Table 2 . The majority of women with CIN2+ $(85 \%)$ had RLU/CO values above 10 , and $1.2 \%, 2.5 \% \%$ and $5.6 \%$ of CIN $2+$ cases were found within RLU/CO intervals of 0.7-2.0, 1.0-2.5 and 0.4-4.0, respectively. The risk for CIN2+ in women within the $0.4-0.69$ and $0.7-0.99$ RLU/CO ranges was $5.6 \%$ and $8.3 \%$, respectively; however, these results represent one woman per range. 
TABLE 2. The distribution of women with a CIN2+/3+ diagnosis and the risk for CIN2+/3+ based on the RLU/CO values of their HPV test results

\begin{tabular}{|c|c|c|c|c|c|}
\hline RLU/CO value & $\begin{array}{c}\mathrm{N} \text { and \% women } \\
\left(\mathrm{N}_{\mathrm{tot}}=594\right)\end{array}$ & $\begin{array}{c}\mathrm{N} \text { and \% CIN2+ } \\
\left(\mathrm{N}_{\text {tot }}=162\right)\end{array}$ & Risk for CIN2+ (\%) & $\begin{array}{c}\mathrm{N} \text { and } \% \text { CIN3+ } \\
\left(\mathrm{N}_{\text {tot }}=114\right)\end{array}$ & $\begin{array}{c}\text { Risk for CIN3+ } \\
(\%)\end{array}$ \\
\hline $0.4 \leq \mathrm{RLU} / \mathrm{CO}<0.7$ & $18(3.0)$ & $1(0.6)$ & 5.6 & $1(0.9)$ & 5.6 \\
\hline $0.7 \leq \mathrm{RLU} / \mathrm{CO}<1.0$ & $12(2.0)$ & $1(0.6)$ & 8.3 & $1(0.9)$ & 8.3 \\
\hline $1.0 \leq \mathrm{RLU} / \mathrm{CO} \leq 2.0$ & $15(2.5)$ & $1(0.6)$ & 6.7 & $1(0.9)$ & 6.7 \\
\hline $2.0<\mathrm{RLU} / \mathrm{CO} \leq 2.5$ & $8(1.3)$ & $3(1.9)$ & 37.5 & $2(1.8)$ & 25.0 \\
\hline $4.0<\mathrm{RLU} / \mathrm{CO} \leq 10.0$ & $26(4.4)$ & $7(4.3)$ & 26.9 & $6(5.3)$ & 23.1 \\
\hline $10.0<$ RLU/CO $\leq 100$ & $112(18.9)$ & $48(29.6)$ & 42.9 & $33(28.9)$ & 29.5 \\
\hline $100<\mathrm{RLU} / \mathrm{CO} \leq 1000$ & 105 (17.7) & $68(42.0)$ & 64.8 & $53(46.5)$ & 50.5 \\
\hline $1000<\mathrm{RLU} / \mathrm{CO}$ & $34(5.7)$ & $22(13.6)$ & 64.7 & $13(11.4)$ & 38.2 \\
\hline
\end{tabular}

$\mathrm{CIN} 2+=$ cervical intraepithelial neoplasia grade 2 or greater; $\mathrm{CIN} 3+=$ cervical intraepithelial neoplasia grade 3 or greater; $\mathrm{N}=$ number; $\mathrm{N}_{\text {tot }}=$ total number

\section{Sensitivity and specificity of the HC2 test for CIN2+}

Calculations of the sensitivity and specificity of $\mathrm{HC} 2$ for CIN2+ at various RLU/CO values are presented in Figure 2. At the threshold recommended by the manufacturer $(\mathrm{RLU} / \mathrm{CO}=1.0)$, the sensitivity was $93.8 \%$ (95\% CI: $90.1-97.5 \%)$, and the specificity was $63.2 \%$ (95\% CI: $58.6-67.6 \%$ ). Increasing or decreasing the threshold in the vicinity of $1.0 \mathrm{RLU} /$ $\mathrm{CO}$ did not significantly improve one value without lowering the other. Increasing the cut-off value from 1.0 to $2.5 \mathrm{RLU} / \mathrm{CO}$ decreased the sensitivity from $93.8 \%$ to $91.4 \%$ (95\% CI: $87.0-95.7 \%$ ), while the specificity increased from $63.2 \%$ to $67.6 \%$ (95\% CI: 63.0-72.0\%). Within the interval of 0.7-2.0 RLU/ $\mathrm{CO}$, the sensitivity remained nearly constant, with 94.4\% (95\% CI: 90.7-97.5\%) at a cut-off value of 0.7 vs. $93.2 \%$ (95\% CI: $89.5-96.9 \%)$ at a cut-off value of 2.0, while the specificity increased from $60.6 \%(95 \%$ CI: $56.0-65.3 \%$ ) to $66.4 \%$ (95\% CI: $61.8-70.8 \%$ ). In the third "grey zone", the sensitivity gradually decreased from $95.1 \%$ (95\% CI: 91.4-98.1\%) at 0.4 RLU/CO to $89.5 \%$ (95\% CI: 84.6-93.8\%) at $4.0 \mathrm{RLU} /$ $\mathrm{CO}$, while the specificity gradually increased from 56.7\% (95\% CI: $51.9-61.3 \%$ ) to $69.4 \%$ (95\% CI: $65.0-$ $73.8 \%)$.

\section{Discussion}

Our results showed that relatively few $\mathrm{HC} 2$ test results fell within the "grey zone" ranges currently used by the Institute of Oncology Ljubljana and those proposed by the manufacturer for PreservCyt specimens (3.7\% and $4.5 \%$, respectively). The "grey

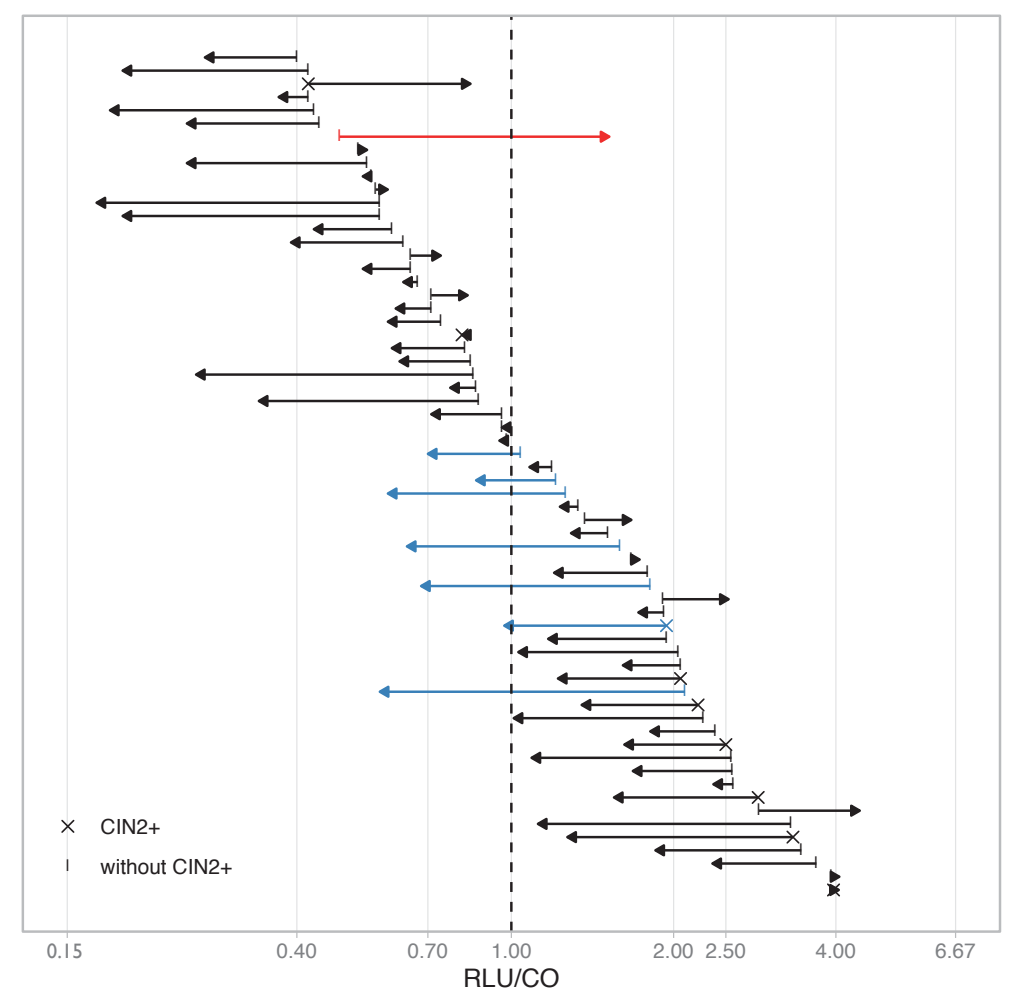

FIGURE 1. Changes in the RLU/CO values after retesting samples within the 0.4-4.0 $\mathrm{RLU} / \mathrm{CO}$ range. The red arrow represents the samples with changes in the results from negative to positive, the blue arrows represent samples with changes in the results from positive to negative, and the black arrows represent samples that retained the original result.

zone" proposed by Seme et al. ${ }^{6}$ was broader and contained $10.8 \%$ of the $\mathrm{HC} 2$ results. The percentages of CIN2+ diagnoses detected within the abovementioned "grey zones" were $1.2 \%, 2.5 \%$ and $5.6 \%$, respectively. Retesting the samples within the broadest "grey zone" investigated did not detect 


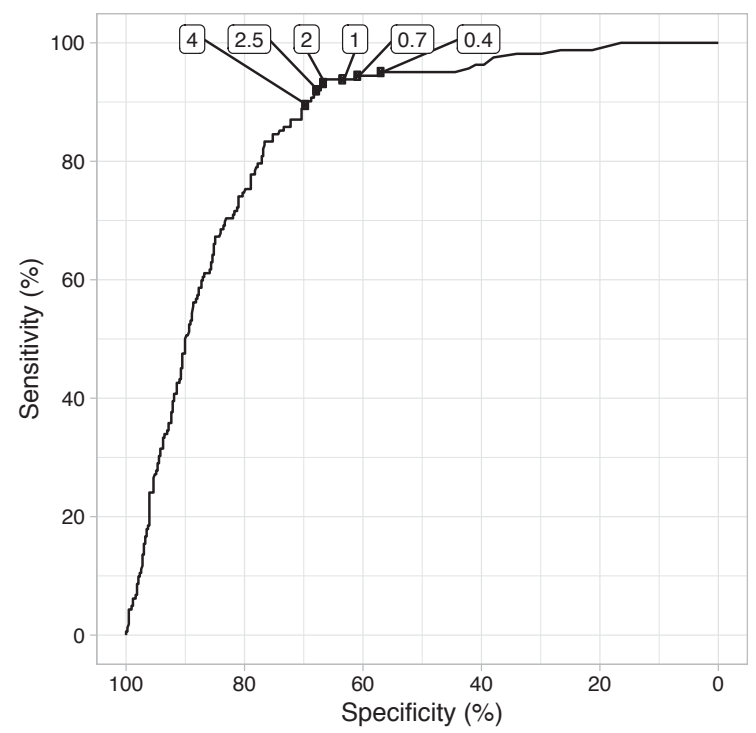

FIGURE 2. ROC curve demonstrating the sensitivity and specificity of the HC2 test for CIN2+ with marked RLU/CO cutoff values that represent the lower and upper borders of the "grey zone" ranges.

additional CIN2+ cases. Calculations of sensitivity and specificity at different RLU/CO values showed that increasing or decreasing the cut-off value within the three "grey zones" did not significantly improve either of the variables.

We have not found any studies that examined the proportion of STM specimens with $\mathrm{HC} 2$ results located in the vicinity of a cut-off value. However, a few studies have reported on the number of $\mathrm{HC} 2$ results found in the "grey zone" interval proposed by the manufacturer (1.0-2.5 RLU/CO) for PreservCyt specimens. ${ }^{9-11}$ Muldrew et al. ${ }^{9}$, Rao et al. ${ }^{10}$ and Knoepp et al..$^{11}$ found $3-5.2 \%$ of their specimens within this "grey zone", which is similar to our result of $4.5 \%$. This finding implies that the frequency of cases with $\mathrm{HC} 2$ results within this "grey zone" is similar, regardless of the medium used for specimen collection (STM or PreservCyt).

From a clinical point of view, the number of CIN2+ cases found within a "grey zone" is much more relevant than the number of $\mathrm{HC} 2$ equivocal results. Among all CIN2+ cases found in our whole study group, only $1.2 \%$ was within $0.7-2.0 \mathrm{RLU} /$ $\mathrm{CO}, 2.5 \%$ was within $1.0-2.5 \mathrm{RLU} / \mathrm{CO}$ and $5.6 \%$ was within 0.4-4.0 RLU/CO. Although several studies have evaluated the proportion of women with CIN2+ diagnoses among those with HC2 results near the cut-off point, it is difficult to compare our results to theirs. The results vary to a moderate extent, and some differences could be attributed to differences in the studied populations and to the definition of the equivocal results. Knoepp et al. ${ }^{12}$, for example, reported $8 \%$ of CIN2+ cases among the whole study population with equivocal HC2 results (1.0-2.5 RLU/CO) and $16.5 \%$ of CIN2+ cases of equivocal HC2 and ASC-US cytology. Origoni et al. ${ }^{7}$ found only $4.6 \%$ of CIN2+ cases in women with ASC-US cytology and HC2 between 1.0 and $10.0 \mathrm{RLU} / \mathrm{CO}$, while LaMere et al. ${ }^{13}$ reported $6.8 \%$ CIN2+ among cases with low-grade cytology and 1.0-3.0 RLU/CO. Interestingly, Elkins et al. $.^{14} \mathrm{dem}-$ onstrated that the clinical relevance of the $\mathrm{HC} 2$ test is age dependent. In two age groups, which together ranged from 15-49 years, these authors found an approximately equal percentage of CIN2+ diagnoses $(6 \%)$ within the "grey zone" of 1.0-2.5 RLU/CO, while there were no CIN2+ cases in the group aged 50 years or more. Despite variations in their results, all authors concluded that women with equivocal HC2 results should be managed as unequivocal positive results.

Since the manufacturer recommends retesting PreservCyt specimens with results within the "grey zone", several authors have already evaluated whether the retesting algorithm is effective. ${ }^{11,}$ 13-16 Some authors have performed one retest ${ }^{15}$, 16, while others have reported two retests ${ }^{13}$, and some retested only those samples where the results were found within the "grey zone" after the first retest ${ }^{11}$ or below 1.0 RLU/CO. ${ }^{14}$ The range of the "grey zone" varied from 0.8 to 3.0 RLU/CO. Some authors have found that the majority of their specimens retained the original positive (87\%-97\%) HC2 result. ${ }^{11,13,14}$ These results are comparable to our results for the STM specimens, which showed that $87.5 \%$ of specimens retained the original HC2 test result after retest, even though our "grey zone" was wider. Ramirez et al. ${ }^{15}$ and de Vries et al. ${ }^{16} \mathrm{ob}-$ tained lower values for specimens that retained original HC2 diagnoses after retest $(64 \%$ and $74 \%$, respectively). Ramirez et al..$^{15}$ was the only study besides ours where the "grey zone" extended below $1.0 \mathrm{RLU} / \mathrm{CO}$, and these authors also found that two results changed from negative to positive. The low percentage of cases that retained positive HC2 results in the study of de Vries ${ }^{16}$ is due to the age of their study population of 50 years or older. All the above-mentioned authors concluded that retest is not necessary because few results change after retest or because retests might be less reliable. For example, Ramirez et al. ${ }^{15}$ mentioned that the amount of sample can influence the results, and viral loads may vary in successive tests.

The expected added value of retesting equivocal HC2 results is a potential increase in the ac- 
curacy of the triage test and a better identification of women who have low-risk for developing CIN2+ lesions since these individuals could be returned to screening or to a less intensive followup. However, an additional reason that speaks against retesting samples within the "grey zones" is the finding that sensitivity and specificity do not change much within these "grey zone" ranges. Calculating the sensitivity of the HC2 test at different RLU/CO values demonstrated that decreasing the cut-off for positivity to the lower border of the "grey zone" range would achieve a small increase in the sensitivity and therefore would not add a higher accuracy of the test. The sensitivity was $95.1 \%$ at a cut-off value of $0.4,94.4 \%$ at the cut-off value of 0.7 and $93.8 \%$ at the cut-off value of 1.0 RLU/CO. Several authors have performed similar studies ${ }^{20-26}$, and a systematic review by Rebolj et al. ${ }^{27}$ concluded that the threshold could be increased to values between 2.0-10.0 RLU/CO without endangering the sensitivity level necessary for screening. This increase would avoid the problem of "grey zones". Compared to our study, the majority of the reported studies have compared the relative values of sensitivity, since HPV-negative women did not undergo colposcopy examinations. Therefore, their calculations did not include the CIN2+ lesions with $\mathrm{HC} 2$ results in the negative part of the RLU/ $\mathrm{CO}$ range since such cases were missed. Thus, their values of sensitivity were higher.

The strength of our study is in reporting results within "grey zones" for STM specimens and investigating two "grey zones" that extended below 1.0 RLU/CO. The results within "grey zones" for STM samples have not been presented before, and most reports included "grey zones" above the proposed cut-off value. The limitation of our study is a small study population, comprising women invited to colposcopy, which showed a higher incidence of CIN2+ lesions. The risk for developing CIN2+ was higher within the investigated "grey zones" (7.4\%, $17.4 \%$ and $14.1 \%$, respectively) than among the general population since the prevalence of the disease was higher in women referred to colposcopy than in the population of women in our study. Therefore, our findings need to be tested on a larger population with the risk for CIN2+ comparable to that of the population where triage is recommended. An additional limitation of our study is the use of residual samples that were stored in the freezer $\left(-30^{\circ} \mathrm{C}\right)$ after the denaturation step for $\mathrm{HC} 2$ retesting. This storage procedure could have caused sample degradation and might have influenced our results. These findings will be important for cervical cancer screening programmes that use the HC2 assay as a primary or triage test and collect cervical specimens in STM. The results will help specify the best protocol for handling STM specimens with results within the "grey zone".

In conclusion, our results show that retesting STM samples within the "grey zones" is not necessary for several reasons. The majority of samples within the "grey zone" retain the original HC2 results after retest. The number of CIN2+ lesions among women with "grey zone" $\mathrm{HC} 2$ results is low. There is limited additional value of the retesting algorithm since sensitivity and specificity of HC2 for CIN2+ do not change much within the "grey zone". Retesting samples with HC2 results in the negative range of the "grey zone" does not increase sensitivity, while retesting in the positive "grey zone" does not add to a less intensive management of women. Women with $\mathrm{HC} 2$ results above 1.0 RLU/CO but within the "grey zone" will be followed in the same manner, regardless of the outcome of the retest. Only women with two negative HC2 results will return to regular screening. Furthermore, according to Slovenian clinical guidelines, the management of women with discordant results between the original test and the repeated HC2 test does not allow women to be returned to screening or to a less intensive follow-up, since at least one additional test is needed before the decision could be made about further management.

\section{References}

1. Ursič Vrščaj M, Rakar S, Možina A, Kobal B, Takač I, Deisinger I, et al. [Guidelines for management of women with cervical precancerous lesions]. [Slovenian]. In: Ursic-Vrscaj M, editor. Ljubljana: Institute of Oncology Ljubljana; 2011.

2. Hybrid Capture 2 (hc2) High Risk HPV DNA test kit [package insert]. Gaithersburg, MD: Digene; 2004.

3. Castle PE, Lorincz AT, Mielzynska-Lohnas I, Scott DR, Glass AG, Sherman $\mathrm{ME}$, et al. Results of human papillomavirus DNA testing with the Hybrid Capture 2 assay are reproducible. J Clin Microbiol 2002; 40: 1088-90. doi: 10.1158/1055-9965.EPI-07-2904

4. Castle PE, Wheeler CM, Solomon D, Schiffman M, Peyton CL; ALTS Group Interlaboratory reliability of Hybrid Capture 2. Am J Clin Pathol 2004; 122: 238-45. doi: 10.1158/1055-9965.EPI-07-2904

5. Carozzi FM, Del Mistro A, Confortini M, Sani C, Puliti D, Trevisan R, et al Reproducibility of HPV DNA testing by Hybrid Capture 2 in a screening setting. Am J Clin Pathol 2005; 124: 716-21. doi: 10.1309/84E5WHJQHK83BGQD

6. Seme K, Fujs K, Kocjan BJ, Poljak M. Resolving repeatedly borderline results of Hybrid Capture 2 HPV DNA Test using polymerase chain reaction and genotyping. J Virol Methods 2006; 134: 252-6. doi: 10.1016/j. jviromet.2005.12.004

7. Origoni M, Carminati G, Sideri M, Clementi M, Rolla S, Candiani M. "Lowgrade positivity" of HPV viral load after atypical squamous cells of undetermined significance (ASC-US) cytology identifies women at low-risk for cervical intraepithelial neoplasia grade 2 and 3. Eur J Gynaecol Oncol 2012; 33: 261-4. PMID: 22873095 
8. de Cremoux P, Coste J, Sastre-Garau X, Thioux M, Bouillac C, Labbe S, et al. Efficiency of the Hybrid Capture 2 HPV DNA test in cervical cancer screening. A study by the French Society of Clinical Cytology. Am J Clin Pathol 2003; 120: 492-9. doi: 10.1309/XFUC-PP6M-5XUA-94B8

9. Muldrew KL, Beqaj SH, Han J, Lum SH, Clinard V, Schultenover SJ, et al. Evaluation of a Digene-recommended algorithm for human papillomavirus low-positive results present in a "retest zone". Am J Clin Pathol 2007; 127: 97-102. doi: 10.1309/4WCPTUV506HLP06P

10. Rao A, Sandri MT, Sideri M, Young S, Sharma A, Behrens C. Comparison of hybrid capture 2 High-Risk HPV results in the low positive range with cobas ${ }^{\circledR}$ HPV Test results from the ATHENA study. J Clin Virol 2013; 58: 161-7. doi: 10.1016/j.jcv.2013.06.041

11. Knoepp SM, Kuebler DL, Wilbur DC. Resolution of equivocal results with the Hybrid Capture II high-risk HPV DNA test: a cytologic/histologic review of 191 cases. Diagn Mol Pathol 2007; 16: 125-9. doi: 10.1097/ PDM.0b013e31805c99ae

12. Knoepp SM, Kuebler DL, Wilbur DC. Correlation between hybrid capture II high-risk human papillomavirus DNA test chemiluminescence intensity from cervical samples with follow-up histologic results: a cytologic/ histologic review of 367 cases. Cancer Cytopathol 2010; 118: 209-17. doi: $10.1002 /$ cncy.20093

13. LaMere BJ, Castle PE, Fetterman B, Poitras N, Stanley M, Shieh J, et al. A study of borderline positive Hybrid Capture 2 tests in the Kaiser Permanente Northern California cervical screening program: evidence against retesting. $J$ Virol Methods 2013; 189: 77-9. doi: 10.1016/j.jviromet.2013.01.011

14. Elkins $C T$, de Vries CE, Stephens J, Suarez AA. Hybrid capture 2 test results after an initial equivocal RLU/CO value are dependent on age. Am J Clin Pathol 2013; 139: 605-10. doi: 10.1309/AJCPARHTB40DTVFV

15. Ramirez-Hidalgo A, Musset-Biarnes M, Vilamala-Muns M, Laso-Perez E, Serrano-Munne L, Alameda-Quitllet F. Hybrid capture 2 high-risk human papillomavirus test: should "grey zone" results justify repeating the test? Anal Quant Cytopathol Histpathol 2013; 35: 152-6. PMID: 24344502

16. de Vries CE, Shen R, Stephens J, Suarez AA. Equivocal and weakly positive hybrid capture 2 test in women aged 50 and older. Diagn Cytopathol 2012; 40: $708-12$. doi: $10.1002 / d c .21710$

17. Ivanus U, Jerman T, Fokter AR, Takac I, Prevodnik VK, Marcec M, et al. Randomised trial of HPV self-sampling among non-attenders in the Slovenian cervical screening programme ZORA: comparing three different screening approaches. Radiol Oncol 2018; 52: 399-412. doi: 10.2478/ raon-2018-0036

18. Kurman RJ, Carcangiu ML, Herrington CS, Young RH, editors. WHO classification of tumours of female reproductive organs. 4th edition. Lyon: International Agency for Research on Cancer; 2014

19. R Core Team. The R Project for Statistical Computing. R: A Language and Environment for Statistical Computing. Vienna: R Foundation for Statistical Computing; 2019. [cited 2019 May 15]. Available at: http://www.R-project. org/.

20. Kotaniemi-Talonen L, Malila N, Nieminen P, Anttila A, Tarkkanen J, Laurila $P$, et al. Test positivity cutoff level of a high risk human papillomavirus test could be increased in routine cervical cancer screening. Int J Cancer 2008; 123: 2902-6. doi: 10.1002/ijc.23839.

21. Ronco G, Giorgi-Rossi P, Carozzi F, Dalla Palma P, Del Mistro A, De Marco L, et al. Human papillomavirus testing and liquid-based cytology in primary screening of women younger than 35 years: results at recruitment for randomised controlled trial. Lancet Oncol 2006; 7: 547-55. doi: 10.1016/ S1470-2045(06)70731-8

22. Ronco G, Segnan N, Giorgi-Rossi P, Zappa M, Casadei GP, Carozzi F, et al. Human papillomavirus testing and liquid-based cytology: results at recruitment from the new technologies for cervical cancer randomized controlled trial. J Nat/ Cancer Inst 2006; 98: 765-74. doi: 10.1093/jnci/djj209

23. Ronco G, Giorgi-Rossi P, Carozzi F, Confortini M, Dalla Palma P, Del Mistro A et al. Results at recruitment from a randomized controlled trial comparing human papillomavirus testing alone with conventional cytology as the primary cervical cancer screening test. J Nat/ Cancer Inst 2008; 100: 492-501. doi: 10.1093/jnci/djn065

24. Sargent A, Bailey A, Turner A, Almonte M, Gilham C, Baysson H, et al Optimal threshold for a positive hybrid capture 2 test for detection of human papillomavirus: data from the ARTISTIC trial. J Clin Microbiol 2010; 48: 554-8. doi: 10.1128/JCM.00896-09
25. Clavel C, Masure M, Bory JP, Putaud I, Mangeonjean C, Lorenzato M, et al. Human papillomavirus testing in primary screening for the detection of high-grade cervical lesions: a study of 7932 women. Br J Cancer 2001; 84: 1616-23. doi: 10.1054/bjoc.2001.1845

26. Rijkaart DC, Coupe VM, van Kemenade FJ, Heideman DA, Hesselink AT, Verweij W, et al. Comparison of Hybrid capture 2 testing at different thresholds with cytology as primary cervical screening test. Br J Cancer 2010; 103: 939-46. doi: $10.1038 /$ sj.bjc.6605869

27. Rebolj M, Bonde J, Njor SH, Lynge E. Human papillomavirus testing in primary cervical screening and the cut-off level for hybrid capture 2 tests: systematic review. BMJ 2011; 23: 342. doi: 10.1136/bmj.d2757 